01,09

\title{
Ab initio моделирование электронной структуры и оптическая спектроскопия соединения ErRhGe
}

\author{
(С) Ю.В. Князев ${ }^{1}$, А.В. Лукоянов ${ }^{1,2}$, Ю.И. Кузьмин ${ }^{1}$, S. Gupta ${ }^{3}$, K.G. Suresh ${ }^{3}$ \\ ${ }^{1}$ Институт фризики металлов им. М.Н. Михеева УрО РАН, \\ Екатеринбург, Россия \\ ${ }^{2}$ Уральский фредеральный университет им. Б.Н. Ельцина, \\ Екатеринбург, Россия \\ ${ }^{3}$ Department of Physics, Indian Institute of Technology Bombay, \\ Mumbai, India \\ E-mail: knyazev@imp.uran.ru \\ (Поступила в Редакцию 21 декабря 2016 г.)
}

Представлены результаты исследования электронной структуры и оптических свойств соединения ErRhGe. В приближении локальной электронной спиновой плотности с поправкой на сильные электронные взаимодействия в $4 f$-оболочке редкоземельного металла (метод LSDA + U) проведены расчеты зонного спектра с учетом спиновой поляризации. В широком интервале длин волн эллипсометрическим методом измерены значения оптических постоянных соединения, определен ряд спектральных и электронных характеристик. Структурные особенности спектра оптической проводимости в области межзонного поглощения света интерпретированы на основе рассчитанной плотности электронных состояний.

Работа выполнена в рамках государственного задания ФАНО России (тема „Электрон“, № 01201463326) при частичной поддержке РФФИ (проект № 17-02-00412). А.В. Лукоянов благодарит за поддержку Правительство РФ (постановление № 211, контракт № 02.А03.21.0006) и стипендиальную программу Президента РФ СП-226.2015.2.

DOI: $10.21883 /$ FTT.2017.07.44582.455

\section{1. Введение}

Тройные эквиатомные соединения типа $R T X$ (где $R$ - редкоземельный металл, $T$ и $X$ - переходные $d$ - и $p$-металлы соответственно) активно исследуются благодаря разнообразию их магнитных и электронных свойств, представляющих фундаментальный и практический интерес. Некоторые композиции таких сплавов, синтезированных и исследуемых в последние годы, обладают гигантскими магниторезистивным и магнитокалорическим эффектами, высокой способностью к абсорбции атомарного водорода, что позволяет рассматривать их в качестве материалов для функциональных и конструкторских приложений (см. обзор [1]). Аномальные магнитотранспортные свойства указанных соединений, как правило, связаны с модификацией их магнитного состояния при приложении внешнего поля и сопровождаются изменением объема или симметрии кристаллической решетки [1-6]. Электронные и тепловые характеристики таких материалов вблизи температур магнитных переходов также испытывают существенные трансформации. Представителем данной серии является соединение ErRhGe, структурные, магнитные и электронные свойства которого исследованы в работах [7-9]. Нейтронографические [7] и рентгеновские [8,9] исследования показали, что данный интерметаллид обладает орторомбической кристаллической структурой типа TiNiSi. При низких температурах соединение последовательно переходит сначала в антиферромагнитное (АФМ) состояние с коллинеарным типом упорядочения магнитных моментов (температура Нееля $T_{N}=10.2 \mathrm{~K}$ ), а затем при $5 \mathrm{~K}-$ в состояние с синусоидальным несоразмерным типом магнитной структуры. Экспериментальные измерения намагниченности, теплоемкости и электросопротивления позволили выявить наличие сильных аномалий вблизи указанных температур [9]. В соединении обнаружены большие величины магнитокалорического эффекта и магнетосопротивления; последнее меняет знак при переходе через $T_{N}$. В настоящей работе мы фокусируем внимание на изучении электронной структуры данного интерметаллида. С этой целью выполнены $a b$ initio расчеты зонного спектра ErRhGe, а также проведены исследования оптических свойств в широком интервале длин волн. Результаты эксперимента обсуждаются с учетом рассчитанных плотностей электронных состояний.

\section{2. Расчет электронной структуры}

Интерметаллическое соединение ErRhGe обладает кристаллической структурой орторомбического TiNiSi-типа с пространственной группой симметрии Рпта. Его электронная структура рассчитывалась в пакете компьютерных программ QUANTUM EXPRESSO [10]. Использовался обменно-корреляционный потенциал в приближении обобщенной градиентной поправки (GGA) в версии РВE [11]. Волновые функции раскладывались по плоским волнам. Взаимодействия между ионами и валентными электронами 


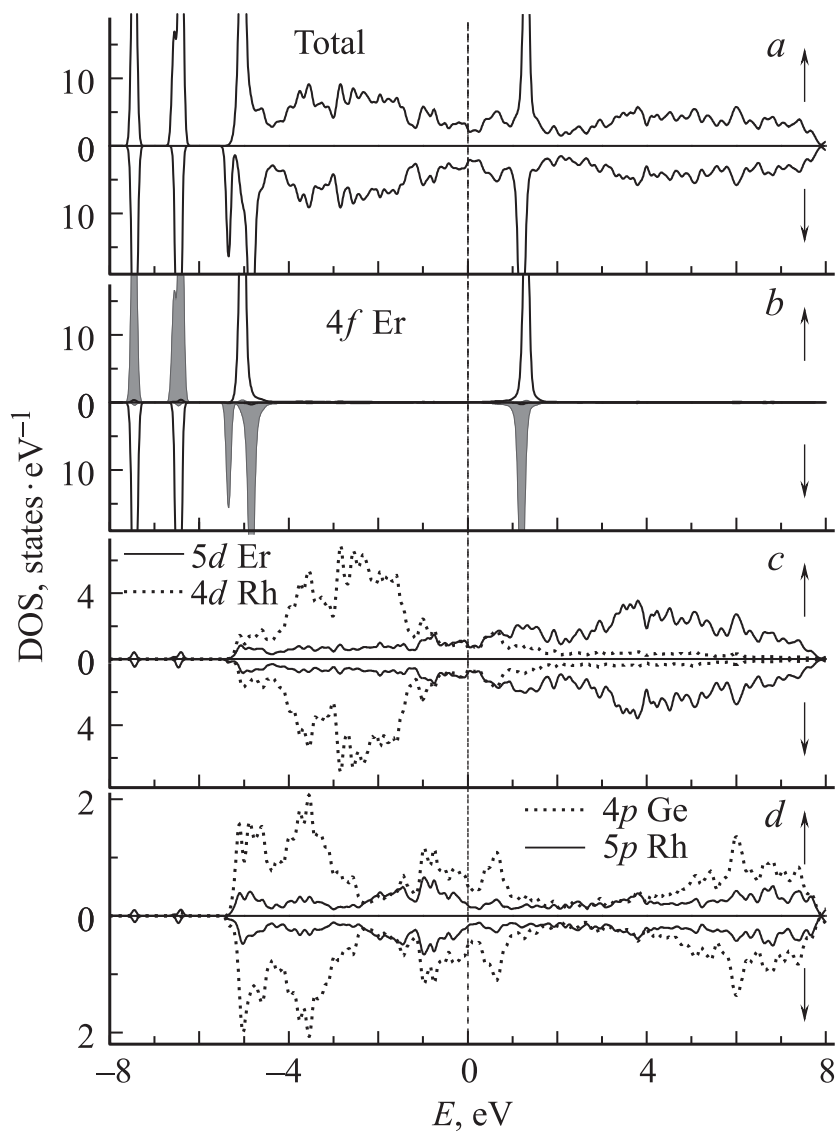

Рис. 1. Полные $(a)$ и парциальные $(b-d)$ плотности электронных состояний антиферромагнитного соединения ErRhGe. Сплошная линия и затемненные области на части $b$ coответствуют атомам $\mathrm{Er}$ с противоположно направленными спиновыми моментами.

учитывались в рамках метода присоединенных плоских волн (PAW). В расчетах были использованы следующие PAW-потенциалы: Er.GGA-PBE-paw-vl.0.UPF [12], Rh.pbespn-kjpaw-ps1.0.2.3.UPF и Ge.pbe-kjpaw.UPF, полученные из программы ATOMPAW [13]. Для достижения самосогласования в расчетах был выбран энергетический предел 60 Rу для плоских волн. Интегрирование в обратном пространстве производилось по сетке из $6 \times 6 \times 6$ $k$-точек. Учет сильных корреляций $4 f$-электронов $\mathrm{Er}$ осуществлялся в рамках метода LSDA + U [14]. Для этого были выбраны следующие величины параметров прямого кулоновского $U$ и обменного хундовского $J$ взаимодействий: $U=4.9 \mathrm{eV}$ и $J=0.7 \mathrm{eV}$. Эти величины дают значение эффективного параметра $U_{\text {eff }}=4.2 \mathrm{eV}$, рекомендованного для $4 f$-состояний $\operatorname{Er}$ в работе [12]. Полученная величина спиновой поляризации $4 f$-электронов эрбия соответствует спиновому моменту $3 \mu_{\mathrm{B}}$ на редкоземельный ион, что согласуется с экспериментальной величиной магнитного момента, полученной с учетом орбитального вклада [9]. В расчетах, принимая во внимание магнитные измерения [9], мы моделировали АФМ-упорядочение магнитных моментов ионов $\mathrm{Er}$ в рамках одной ячейки. Ионы других металлов в расчетах получились немагнитными.

На рис. 1, $а$ представлена полная плотность электронных состояний соединения ErRhGe для двух спиновых проекций $(\uparrow$ и $\downarrow)$. На рис. $1, b-d$ приведены парциальные плотности для $4 f$ - и $5 d$-электронов $\mathrm{Er}, 4 d$ - и $5 p$-электронов $\mathrm{Rh}$ и $4 p$-электронов $\mathrm{Ge}$. Другие плотности состояний на рисунке не приводятся ввиду их малых значений. Узкие интенсивные пики, расположенные в интервале на $4.5-7.5 \mathrm{eV}$ ниже уровня Ферми $E_{\mathrm{F}}$ и вблизи значения $1.3 \mathrm{eV}$ выше $E_{\mathrm{F}}$ (рис. $1, b$ ), образованы заполненными и свободными $4 f$-состояниями эрбия соответственно. Два различных типа ионов эрбия, ответственные за АФМ-упорядочение магнитных моментов, дают пики при одинаковых энергиях, но в противоположных спиновых проекциях. Энергетический диапазон, разделяющий эти состояния, определяется величиной параметра $U$ для $4 f$-оболочки Er. Парциальные плотности состояний для $d$ - и $p$-электронов, расположенные по обе стороны от $E_{\mathrm{F}}$ (от -5 до $\left.8 \mathrm{eV}\right)$, ввиду отсутствия поляризации данных состояний в расчете имеют многопиковую структуру, практически идентичную по величине и форме в обеих спиновых подзонах.

\section{3. Результаты эксперимента и обсуждение}

Исследуемое поликристаллическое соединение ErRhGe выплавлено в дуговой печи в атмосфере очищенного аргона из составных элементов, взятых в стехиометрических пропорциях. Слиток переплавлялся несколько раз, чтобы обеспечить равномерное перемешивание компонентов. Для достижения гомогенности образец подвергался отжигу при $\sim 800^{\circ} \mathrm{C}$ в течение восьми суток. Рентгеноструктурные исследования проводились на дифрактометре X-Pert PRO в монохроматическом $\mathrm{Cu} K_{\alpha}$-излучении $(\lambda=1.54 \AA)$ при комнатной температуре. Методом полнопрофильного анализа Ритвельда установлено, что полученное соединение обладает однофазной орторомбической структурой типа TiNiSi. Параметры кристаллической решетки составляют $a=6.79 \AA, b=4.24 \AA, c=7.49 \AA$. Данные величины использовались при вычислении электронной структуры соединения.

Спектральные свойства ErRhGe исследовались при комнатной температуре в области длин волн $\lambda=0.22-15 \mu \mathrm{m} \quad(E=0.083-5.64 \mathrm{eV})$. Образец имел зеркальную поверхность четырнадцатого класса, приготовленную с помощью механической полировки. Оптические постоянные соединения - показатели преломления и поглощения - измерены эллипсометрическим методом, основанным на определении отношения амплитуд и разности фаз отраженного света $s$ и $p$-поляризаций. По значениям оптических постоянных рассчитаны компоненты комплексной диэлектрической проницаемости $\varepsilon$ и отражательная способность $R$. Энергетические зависимости действительной части 


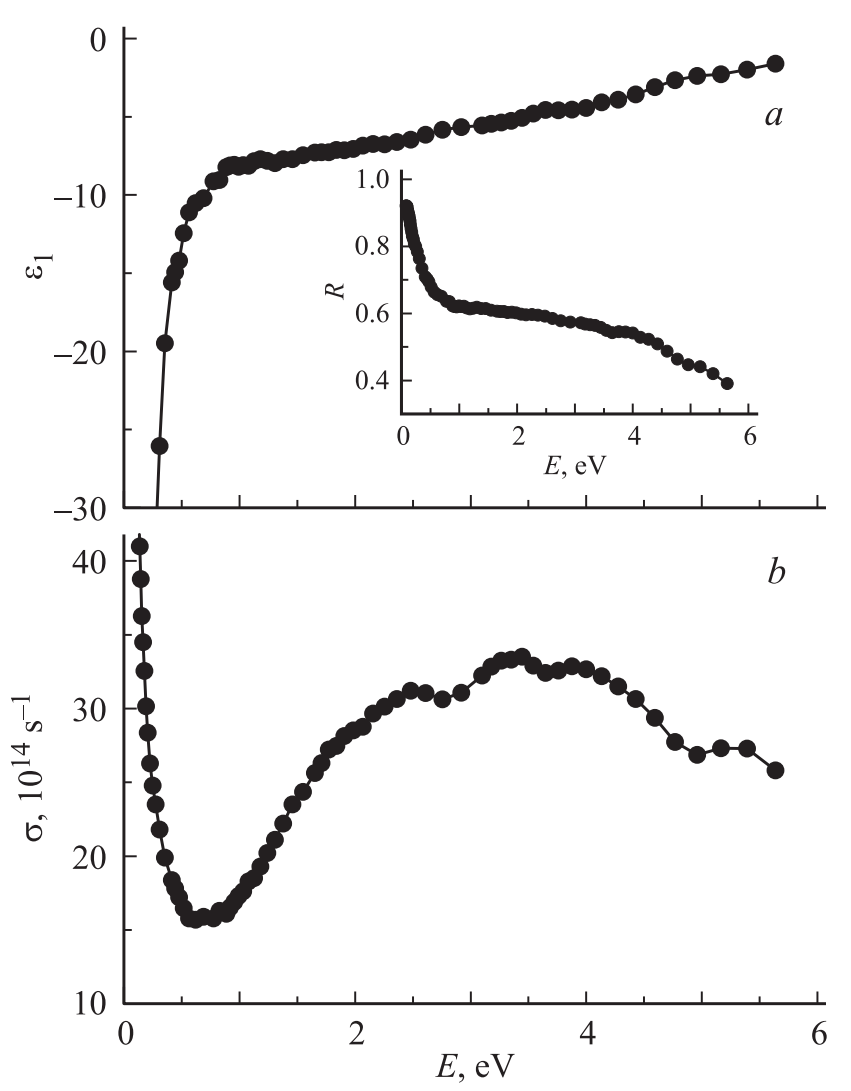

Рис. 2. Действительная часть диэлектрической проницаемости $\varepsilon_{1}$ и отражательная способность $R$ (на вставке) $(a)$, а также оптическая проводимость $\sigma(b)$ соединения ErRhGe.

диэлектрической проницаемости $\varepsilon_{1}$ и $R$ представлены на рис. 2,a. Их поведение типично для материалов с металлическим типом проводимости: значения $\varepsilon_{1}$ отрицательны во всем интервале длин волн, а величина $R$ с уменьшением частоты света стремится к единице. Через мнимую часть диэлектрической проницаемости $\varepsilon_{2}$ выражается оптическая проводимость $\sigma(\omega)=\varepsilon_{2} \omega / 4 \pi(\omega-$ циклическая частота света) - наиболее чувствительный спектральный параметр, характеризующий частотную зависимость и интенсивность оптического отклика среды (рис. 2, $b$ ). В структуре экспериментального спектра $\sigma(\omega)$ отчетливо проявляются два энергетических интервала, относящиеся к различным механизмам возбуждения электронов электромагнитной волной. В низкоэнергетической области $(E \lesssim 0.5 \mathrm{eV})$ на кривой $\sigma(\omega)$ наблюдается монотонный спад, частотная зависимость которого $\left(\sigma \sim \omega^{-2}\right)$ свидетельствует о доминировании внутризонного механизма. Трактовка оптических свойств в этом диапазоне спектра основана на линейном уравнении движения электронов проводимости в переменном поле электромагнитной волны и приводит к классическим соотношениям Друде, по которым можно определить характеристики электронов проводимости: релаксационную $\gamma$ и плазменную $\omega_{p}$ частоты. Частота релаксации $\gamma=\varepsilon_{2} \omega / \varepsilon_{1}$ аддитивно учитывает все виды рассеяния электронов при взаимодействии со световой волной, а плазменная частота $\omega_{p}^{2}=4 \pi N e^{2} / m(N, e$ и $m$ - концентрация, заряд и масса электрона) характеризует коллективные осцилляции валентных электронов. В инфракрасной области спектра выше $10 \mu \mathrm{m}$ эти параметры не зависят от частоты света и принимают значения $\gamma=2.5 \cdot 10^{14} \mathrm{~s}^{-1}, \omega_{p}=4.7 \cdot 10^{15} \mathrm{~s}^{-1}$.

$\mathrm{C}$ ростом энергии фотонов вид частотной дисперсии оптической проводимости свидетельствует о том, что при $E \gtrsim 0.5 \mathrm{eV}$ определяющим является механизм квантовых электронных переходов. Низкоэнергетический спад сменяется широкой полосой межзонного поглощения, в структуре которой проявляется ряд максимумов и минимумов. Форма данной абсорбционной полосы, как правило, индивидуальна для каждого материала и зависит от строения его энергетического спектра. Характер

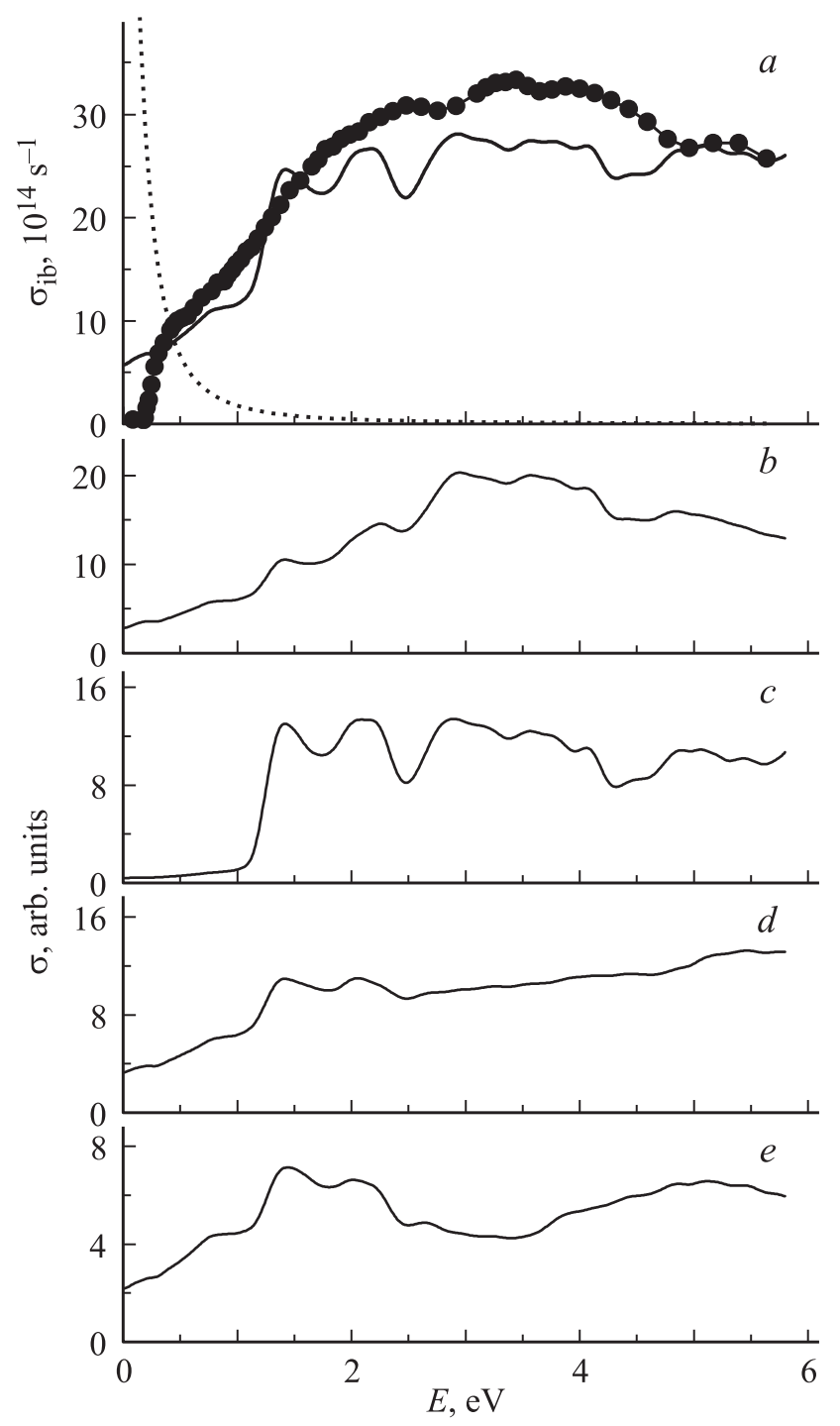

Рис. 3. Спектр межзонной оптической проводимости соединения ErRhGe $(a)$ : точки - эксперимент, сплошная кривая расчет из полной плотности состояний, пунктир - друдевский вклад. $b-e-$ рассчитанные парциальные вклады от межзонных переходов с участием $\mathrm{Rh} 4 d$-, $\mathrm{Er} 4 f$-, $5 d$ - и $\mathrm{Ge} 4 p$-электронов соответственно. 
поведения $\sigma(\omega)$ в этой области энергий определяется суперпозицией вкладов от квантовых переходов в двух спиновых подсистемах между электронными состояниями по обе стороны от $E_{\mathrm{F}}$. Почти симметричный характер зависимостей $N(E)$ для обеих спин-поляризованных зон приводит к тому, что соответствующие вклады в оптическую проводимость сопоставимы по величине. Для объяснения природы формирования межзонного поглощения в ErRhGe представляет интерес сопоставить экспериментальный спектр оптической проводимости с соответствующей теоретической зависимостью, вычисленной из полной плотности электронных состояний $N(E)$. Такое сравнение представлено на рис. 3, где точками показана экспериментальная кривая межзонной проводимости $\sigma_{\mathrm{ib}}(\omega)$, полученная вычитанием друдевского вклада из измеренной зависимости. Спектр теоретической межзонной проводимости $\sigma_{\mathrm{ib}}(\omega)$ рассчитан по методу [15], в соответствии с которым оптическая проводимость в области межзонных переходов определяется свертками $N(E)$ ниже и выше $E_{\mathrm{F}}$. Расчет носит качественный характер, так как выполнен при аппроксимации матричных элементов переходов постоянными значениями, а также при равной вероятности прямых и непрямых переходов, происходящих соответственно с сохранением и изменением квазиимпульса. Сравнение показывает, что, несмотря на отсутствие полной корреляции в деталях тонкой структуры, теоретическая зависимость $\sigma_{\mathrm{ib}}(\omega)$ в целом воспроизводит энергетическое положение и протяженность наблюдаемой полосы поглощения. Экспериментальный спектр является более гладким, чему способствует наложение парциальных вкладов в оптическую проводимость от большого числа электронных переходов с разным временем жизни возбужденного состояния. К сглаживанию спектра, как правило, приводит также экспериментальный фактор, связанный с приготовлением отражающей поверхности.

На рис. $3, b-e$ представлены также результаты расчета наиболее значительных по величине парциальных вкладов в межзонное поглощение, связанных с конкретными электронными состояниями. Видно, что таковыми являются вклады в $\sigma_{\mathrm{ib}}(\omega)$ от квантовых переходов с участием $4 d$-электронов $\mathrm{Rh}$, а также $4 f$ - и $5 d$-электронов $\mathrm{Er}$. Данное обстоятельство указывает на определяющую роль электронов указанных типов в формировании оптического поглощения в области фундаментальной полосы. В соответствии с расчетом зонной структуры соединения ErRhGe (рис. 1) природа этой полосы объясняется главным образом электронными переходами с гибридизированных $\mathrm{Er} 5 d$-, Rh $4 d$ - и Ge $4 p$-зон, расположенных в интервале на $0-5 \mathrm{eV}$ ниже $E_{\mathrm{F}}$, в $4 f$-зону, локализованную вблизи $1 \mathrm{eV}$ выше $E_{\mathrm{F}}$. Отметим, что форма рассчитанной суммарной кривой межзонной проводимости качественно соответствует спектральным профилям парциальных вкладов от $\mathrm{Rh} 4 d$ - и $\operatorname{Er} 4 f$-электронных состояний. При этом вклады от переходов с участием $\mathrm{Er}$ $5 d$ - и Ge $4 p$-электронов характеризуются менее выразительной структурой.

\section{4. Заключение}

Выполнены исследования электронной структуры и оптических свойств интерметаллического соединения ErRhGe. В самосогласованных расчетах, проведенных методом LDSA + U с учетом сильных межэлектронных взаимодействий в $4 f$-оболочке $\mathrm{Er}$, получены полные и парциальные плотности электронных состояний, определены основные параметры зонного спектра для различных типов электронов. Эллипсометрическим методом измерены оптические постоянные, по которым рассчитаны диэлектрические функции, характеризующие оптический отклик материала. С учетом результатов расчета идентифицирована природа электронных состояний, формирующих спектр оптической проводимости в области квантовых электронных переходов. Установлено качественное соответствие экспериментальной и теоретической частотных зависимостей межзонной оптической проводимости.

\section{Список литературы}

[1] S. Gupta, K.G. Suresh. J. Alloys Compd. 618, 562 (2015).

[2] W. Bażela, M. Hofmann, S. Baran, B. Penc, A. Szytuła, A. Zygmunt. Acta Phys. Pol. A 97, 819 (2000).

[3] S. Gupta, K.G. Suresh, A.K. Nigam, Y. Mudryk, D. Paudyal, V.K. Pecharsky, K.A. Gschneidner, jr. J. Alloys Compd. 613, 280 (2014).

[4] J. Gurgul, K. Łatka, A.W. Pacyna, S.C. Peter, R. Pöttgen. Intermetallics 46, 56 (2014).

[5] S. Gupta, K.G. Suresh. J. Magn. Magn. Mater. 391, 151 (2015).

[6] S. Gupta, K.G. Suresh, A.K. Nigam. J. Alloys Compd. 586, 600 (2014).

[7] W. Bażela, M. Hofmann, B. Penc, A. Szytuła. J. Phys.: Condens. Matter 10, 2233 (1998).

[8] A.V. Morozkin, Yu.D. Seropegin, I.A. Sviridov. J. Alloys Compd. 270, L4 (1998).

[9] S. Gupta, K.G. Suresh, A.K. Nigam, A.V. Lukoyanov. J. Alloys Compd. 640, 56 (2015).

[10] P. Giannozzi, S. Baroni, N. Bonini, M. Calandra, R. Car, C. Cavazzoni, D. Ceresoli, G.L. Chiarotti, M. Cococcioni, I. Dabo, A. Dal Corso, S. de Gironcoli, S. Fabris, G. Fratesi, R. Gebauer, U. Gerstmann, C. Gougoussis, A. Kokalj, M. Lazzeri, L. Martin-Samos, N. Marzari, F. Mauri, R. Mazzarello, S. Paolini, A. Pasquarello, L. Paulatto, C. Sbraccia, S. Scandolo, G. Sclauzero, A.P. Seitsonen, A. Smogunov, P. Umari, R.M. Wentzcovitch. J. Phys.: Condens. Matter 21, 395502 (2009).

[11] J.P. Perdew, K. Burke, M. Ernzerhof. Phys. Rev. Lett. 77, 3865 (1996).

[12] M. Topsakal, R.M. Wentzcovitch. Comput. Mater. Sci. 95, 263 (2014).

[13] N.A.W. Holzwarth, A.R. Tackett, G.E. Matthews. Comput. Phys. Commun. 135, 329 (2001).

[14] V.I. Anisimov, F. Aryasetiawan, A.I. Lichtenstein. J. Phys.: Condens. Matter 9, 767 (1997).

[15] Yu.V. Knyazev, A.V. Lukoyanov, Yu.I. Kuz'min, A.G. Kuchin, I.A. Nekrasov. Phys. Rev. B 73, 094410 (2006). 